\title{
Avaliação de Extratos Vegetais como Indicadores Ácido-Base Empregando Titulação Espectrofotométrica em Fluxo Contínuo
}

\section{Jonas A. Vieira, Lourival C. de Faria \& Wesson Guimarães}

Estudo de extratos de frutos de jaboticabeira (Myrciaria cauliflora) e pétalas de flor de picão (Bidens gardneri) como indicadores ácido-base, empregando titulação espectrofotométrica por injeção em fluxo mono canal. O potencial dos extratos como indicador ácido base foi avaliado com base na determinação da acidez em amostras de refrigerantes, suco de abacaxi e vinagre. Na comparação dos resultados por titulação visual, aplicando o teste-t pareado, não foi observada diferença significativa a um nível de $95 \%$ de confiança. O sistema propiciou uma frequência, de 30 determinações por hora e precisão $3,3 \%,(n=10)$

Palavras-chave: extratos indicadores; titulação espectrofotométrica; sistema em fluxo.

Study extracts of Myrciaria cauliflora fruits and petals of Bidens gardneri were used with success as acid base indicators in spectrofotometric titration, employing flow system a single pumping channel to propel sample and reagent solutions. Profitable features such as an analytical throughput of 30 sample determination per hour, relative standard deviation (r.s.d.) of 3,3\% $(\mathrm{n}=10)$. By applying the paired t-test no significant difference at $95 \%$ confidence level was observed between the results obtained with the proposed system end those of the visual titration.

Keywords: indicators extract; spectrophotometric titration; flow system. 


\section{Introdução}

Na titulação por reação de neutralização, o ponto final é analisado com base na mudança de cor do indicador, quando o $\mathrm{pH}$ passa de um meio ácido para alcalino ou vice versa ${ }^{1}$. Isto torna possível a determinação da concentração do ácido em função da concentração padronizada de uma solução alcalina ${ }^{2}$. A automação do método de titulação química em fluxo é contemporâneo com os primeiros sistemas em fluxo. Na década de 50 foi desenvolvido o primeiro titulador automático baseado em sistema de fluxo contínuo ${ }^{3}$, para a determinação de mercaptanas em amostras de gasolina. Posteriormente, esse processo de automação foi adaptado para titulação em sistema de Análise por Injeção em Fluxo (FIA), usado na determinação de ácido clorídrico com hidróxido de sódio ${ }^{4}$. Desde então, foram desenvolvidos outros trabalhos voltados para titulação espectrofotométrica ${ }^{5-11}$.

Os sistemas em fluxo tem sido implementados com quase todas as técnicas de deteç̧ão química, dentre as quais, a espectrofotometria de absorção molecular tem sido uma das mais usada12-16. Assim como os sistemas em fluxo, a titulação espectrofotométrica teve um avanço significativo, constituindo alternativas viáveis para determinações de diferentes espécies químicas, com baixo custo de instrumentação e de análise ${ }^{5-11}$. Em razão da predominância de um grande número de complexos coloridos e/ou reações, com desenvolvimento de $\operatorname{cor}^{17}$, sua aplicação tornou abrangente a diferentes tipos de reações ${ }^{18}$, com destaque para a determinação de acidez em diferentes matrizes ${ }^{19-21,7,9,14,15}$. Neste contexto, tem sido experimentado o uso de extratos de plantas como indicadores, obtendo resultados satisfatórios, principalmente, considerando a facilidade de obtenção dos extratos, bem como, a riqueza da nossa flora ${ }^{22}$.

Nas determinações espectrofotométricas por sistema em fluxo, na avaliação da sensibilidade do método, pode ser considerada a altura ou a largura do sinal transiente, que possibilita construir a curva de calibração e, evidentemente identificar as concentrações dos analitos nas amostras. Sendo que nas titulações espectrofotométricas, predomina a largura do sinal transiente na meia altura do registro ${ }^{4}$.

Neste trabalho avaliou-se dois extratos vegetais quanto ao seu uso como indicador nas reações de neutralização. Os extratos foram obtidos das cascas de jabuticaba (Myrciaria cauliflora), e das pétalas de flores de picão (Bidens gardneri), os estudos foram realizados empregando-se titulação espectrofotométrica por sistema de injeção em fluxo contínuo.

\section{Parte experimental}

\section{EQUIPAMENTOS E ACESSÓRIOS}

O sistema em fluxo foi constituído com uma bomba peristáltica IPC8 da ISMATEC equipada com tubos de Tygon, um injetor automático B-332 da MICRONAL, uma câmara de mistura com volume interno de $1,5 \mathrm{~mL}$, um espectrofotômetro B-330 da FEMTO, equipado com uma cela de fluxo com volume interno de $180 \mu \mathrm{L}$ e caminho óptico de $10 \mathrm{~mm}$, e um registrador potenciométrico RB-101 da ECB Ltda, um agitador magnético CBT da FANEM, Micro pipetas de precisão (Gilson Pipette), modelos P2, P20, P100, P200 e P1000.

\section{REAGENTES E SOLUÇÕES}

As soluções padrão de calibração de $\mathrm{HCl}$, na faixa de concentração de $1,0 \times 10^{-2}$ a $1,0 \times 10^{-1}$ molL-1, foram preparadas por diluição de uma solução estoque de $4 \mathrm{~mol}$ $\mathrm{L}^{-1}$. As soluções de $\mathrm{NaOH}$, nas concentrações de $8,3 \mathrm{x}$ $10^{-4}$ e $8,6 \times 10^{-3} \mathrm{molL}^{-1}$, foram preparadas a partir de uma solução estoque de $12 \mathrm{molL}^{-1}$. Solução de Fenolftaleína $1 \%$ e os extratos de jabuticaba e de picão, foram utilizados como indicadores em titulação de reação de neutralização.

\section{PREPARAÇÃO DOS EXTRATOS}

$\mathrm{O}$ extrato de picão foi obtido pela imersão de $50 \mathrm{~g}$ de pétalas de suas flores em $500 \mathrm{~mL}$ de etanol $96 \%$, sob agitação por 2 horas. $\mathrm{O}$ extrato dos frutos de jabuticaba foi obtido pelo mesmo procedimento, no entanto foram usados $300 \mathrm{~g}$ de material vegetal. Posteriormente, os extratos foram filtrados, cujas soluções obtidas, tiveram os volume reduzido a aproximadamente $30 \mathrm{~mL}$. A redução do volume dos extração foi efetuada por destilação a pressão reduzida a uma temperatura de $55^{\circ} \mathrm{C}$.

Os extratos foram transferidos para frascos escuro, e conservado em geladeira em torno de $10^{\circ} \mathrm{C}$. 


\section{SISTEMA DE ANÁLISE POR INJEÇÃO EM FLUXO}

O módulo de análise do sistema em fluxo, construído para a titulação espectrofotométrica é mostrado na Figura 1. Os estudos foram realizados usando um percurso analítico de $20 \mathrm{~cm}$, sendo $10 \mathrm{~cm}$ do injetor até a câmara de mistura e $10 \mathrm{~cm}$ desta à cela de fluxo do espectrofotômetro e vazão de bombeamento de $1,2 \mathrm{~mL}$ $\min -1$.

Alíquotas com volume de $200 \mu \mathrm{L}$ das soluções das amostras foram introduzidas no sistema em fluxo no ponto A, sendo transportado pela solução de $\mathrm{NaOH}$, contendo uma quantidade fixa de Fenolftaleína ou de um dos extratos em estudo, até a câmara de dispersão, onde ocorria a homogeneização das soluções reagentes.

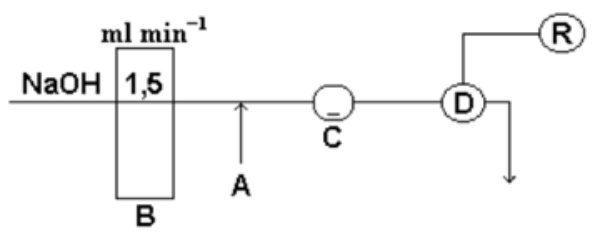

Figura 1: Diagrama do sistema em fluxo. $\mathrm{A}$ = introdução da solução da amostra; $\mathrm{B}=$ bomba peristáltica; $\mathrm{C}=$ câmara de dispersão contendo uma barra magnética; $\mathrm{D}=$ detector; $\mathrm{R}=$ registrador potenciométrico.

Foram conduzidas titulações de $\mathrm{HCl}$ e de amostras de refrigerantes, suco de abacaxi e vinagre com solução padronizada de $\mathrm{NaOH}$. Os experimentos foram efetuados usando-se os extratos naturais bruto e Fenolftaleína, com o objetivo de comparar os resultados. Os estudos foram repetidos pelo método de titulação visual, usado como método de referência.

\section{Resultados e discussão}

Inicialmente avaliou-se a região espectral de maior sensibilidade, para cada extrato usado como indicador, conforme representado nos gráficos da Figura 2. Para o extrato de jabuticaba, foi observada uma maior sensibilidade entre $480-500 \mathrm{~nm}$ e para o extrato de picão, a maior sensibilidade foi observada entre 510 $530 \mathrm{~nm}$. Portanto, fixou-se os comprimentos de onda em 490 e $520 \mathrm{~nm}$, considerando-se os valores intermediários à região de maior sensibilidade para os dois indicadores, respectivamente.

No extrato de jabuticaba, foi observada uma coloração amarelo esverdeado em meio alcalino, incolor em torno de pH 7 e azul em meio ácido, conforme ilustrado na Figura 3 , cujo o intervalo de $\mathrm{pH}$ no ponto de viragem foi 1,73 . No extrato de picão, observou-se uma cor vermelho em meio ácido e amarelo claro em meio alcalino, onde a mudança da cor ocorre em torno de $\mathrm{pH} 7$ (Figura 3), o intervalo de $\mathrm{pH}$ no ponto de viragem foi 2,01. É importante enfatizar que esse comportamento é predominante das substâncias antocianinas $^{23,24}$.

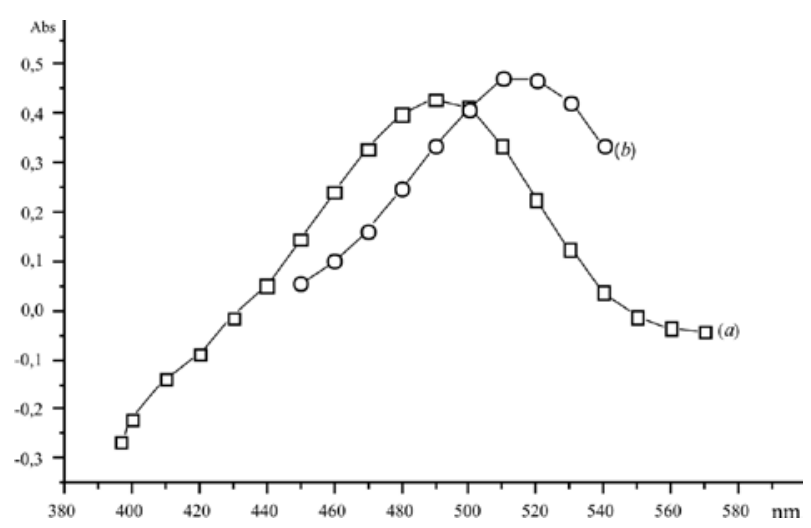

Figura 2: Avaliação da região espectral para os dois extratos em estudo. Testes efetuados usando $\mathrm{NaOH} 0,1 \mathrm{molL}^{-1}, 6$ gotas de indicador em 100 $\mathrm{mL}$ de solução: (a) extrato de jabuticaba, (b) extrato de picão.

Foi observado que a quantidade do indicador propiciava uma variação na sensibilidade relativa a altura do sinal analítico, conforme representado na Figura 4. Os sinais $\mathrm{S}_{1}$ e $\mathrm{S}_{2}$ foram obtidos em função da intensidade de cor, referente a uma e duas gotas de indicador em $50 \mathrm{~mL}$ de $\mathrm{NaOH}$. Observa-se que para as mesmas concentrações do reagente, houve um aumento significativo na absorbância em relação à altura do sinal.

Os resultados obtidos foram: Fenolftaleína $\mathrm{S}_{1}=0,294$ e $\mathrm{S}_{2}=0,503$, extratos de picão $\mathrm{S}_{1}=0,255$ e $\mathrm{S}_{2}=0,366$ e de jabuticaba $S_{1}=0,196$ e $S_{2}=0,255$. No entanto, para as mesmas concentrações dos reagentes a largura do sinal analítico manteve-se constante, o que significa que a variação na quantidade do indicador, não interfere na sensibilidade com relação a largura do sinal transiente. Os teste foram realizados titulando-se uma solução de $\mathrm{NaOH} 8,6 \times 10^{-3}$ molL $^{1}$ com HCl 1,0 x 10-1 molL̃1.

Portanto, ao invés de usar a altura do sinal como parâmetro de avaliação dos resultados, as avaliações 


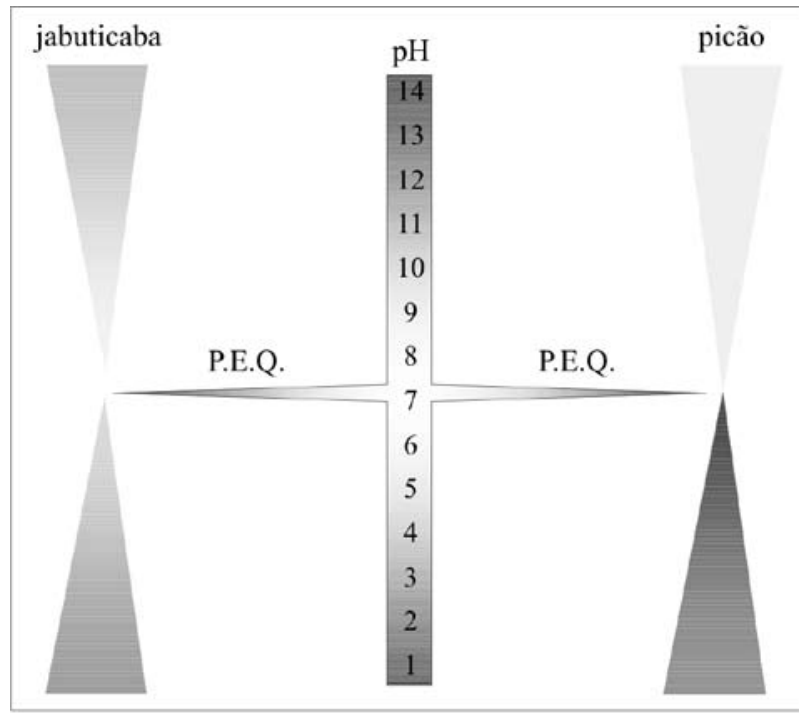

Figura 3: Características das cores dos indicadores em função da variação do $\mathrm{pH}$.

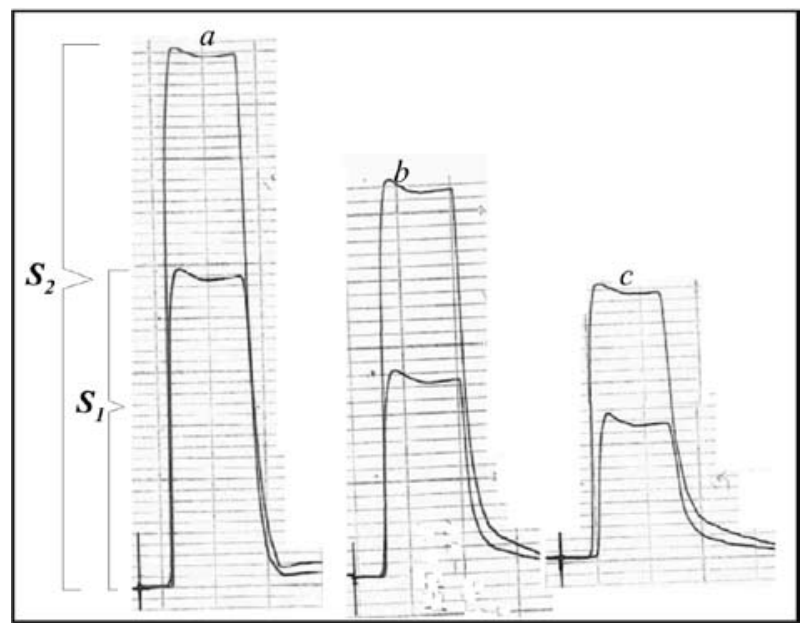

Figura 4: Efeito da quantidade de indicador na sensibilidade do sinal analítico. Soluções: titulada, $\mathrm{NaOH} 8,6 \times 10^{-3}$ molL-1; titulante, $\mathrm{HCl} 1,0$ x $10^{-1} \mathrm{molL}^{-1}$; volume de $\mathrm{HCl}=200 \mu \mathrm{L} . \mathrm{S}_{1}$ e S $_{2}=$ sinais correspondentes a uma e duas gotas de indicador em $50 \mathrm{ml}$ de $\mathrm{NaOH}$. Os registros (a), (b) e (c) são referentes aos indicadores fenolftaleína, extratos picão e de jabuticaba, respectivamente.

foram efetuadas com base na largura dos registros, conforme representado na Figura 5. Sendo que os resultados foram obtidos, usando extrato de picão (a) e extrato de jabuticaba (b).

$\mathrm{Na}$ Figura 5, fica claro a variação do tempo de residência, em função da diferença de concentração das soluções de $\mathrm{HCl}$. Isto significa que, mantendo-

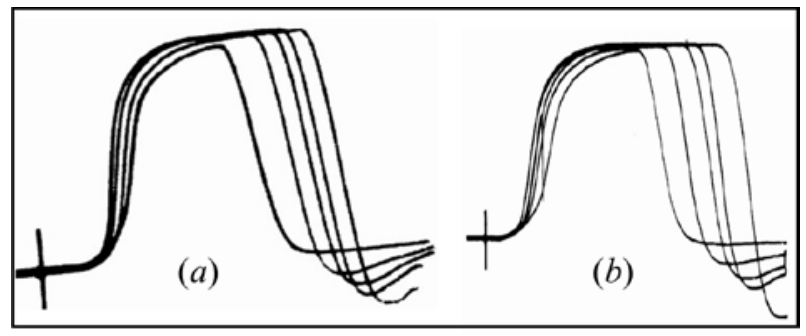

Figura 5: Característica dos registros referentes a titulação espectrofotmétrica em fluxo contínuo. Titulação de uma solução de $\mathrm{NaOH} 0,1 \mathrm{molL}^{-1}$ com soluções de $\mathrm{HCl}$ nas concentrações da esquerda para a direita 0,$2 ; 0,4 ; 0,6 ; 0,7$ e $0,9 \mathrm{molL}^{-1}$.

se constante a concentração da solução de $\mathrm{NaOH}$ e, variando-se a concentração da solução de $\mathrm{HCl}$, resulta num aumento proporcional do tempo de residência, que é caracterizado por um aumento na largura do sinal. Esse parâmetro possibilita a construção de curva de calibração analítica. Devido dificuldades na definição da largura exata do sinal na linha de base, considerou-se a largura na meia altura dos registros.

A avaliação dos extratos de jabuticaba e de picão como indicadores ácido-base, empregando titulação espectrofotométrica em fluxo contínuo, apresentou um desempenho semelhante ao do indicador Fenolftaleína, conforme representado na Figura 6. As curvas de calibração $(a, b, c)$, foram obtidas usando o indicador Fenolftaleína, extrato picão e de jabuticaba, respectivamente. A faixa de concentração linear foi de $1,0 \times 10^{-2}$ a $1,0 \times 10^{-1}$ molL $^{1}$ em HCl, utilizando-se uma solução de $\mathrm{NaOH} 10^{-3}$ mol: ${ }^{1}$. Observa-se que a relação entre a largura dos sinais analíticos e as concentrações de $\mathrm{HCl}$ seguem uma equação de primeira ordem, conforme descritas na Figura 6. A faixa de concentração linear dos resultados usando os extratos naturais brutos, foi semelhante à obtida usando o indicador Fenolftaleína, o que demonstra a viabilidade do uso desses extratos como indicadores.

A viabilidade do processo de titulação espectrofotométrica usando os extratos de jabuticaba e picão, foi avaliada titulando-se uma solução de $\mathrm{NaOH}$ $8,3 \times 10^{-4} \mathrm{molL}^{-1}$ com soluções de $\mathrm{HCl}$ na faixa de $0,1 \mathrm{a}$ 1,0 molL $^{-1}$. bem como a determinação da acidez total de amostras de refrigerantes, suco de abacaxi e vinagre.

As condições de otimização propiciou uma frequência analítica de 30 determinações por hora, precisão de 


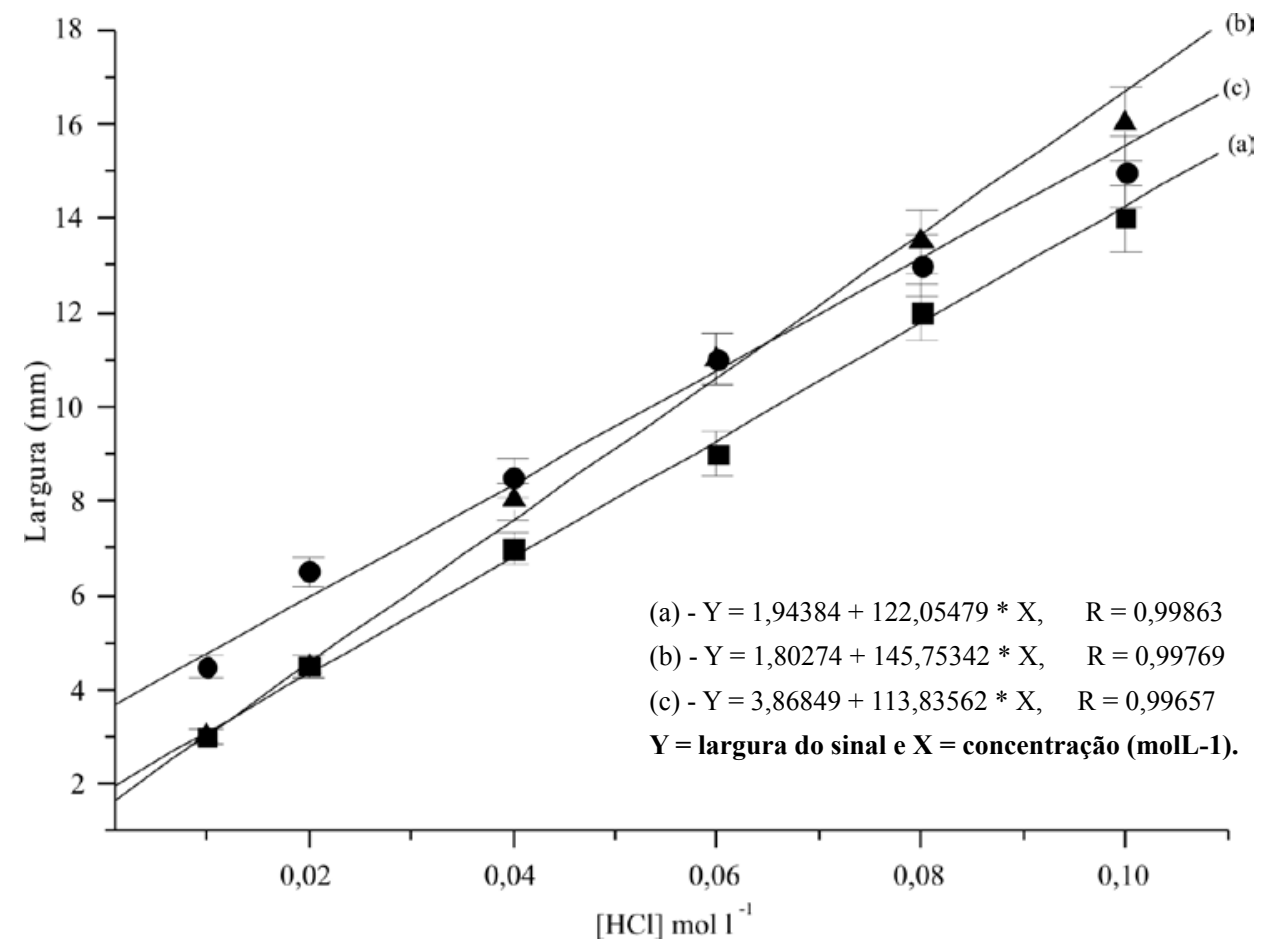

Figura 6: Avaliação do desempenho do sistema em fluxo para titulação espectrofotométrica. As curvas (a), (b) e (c) correspondem aos indicadores fenolftaleína, extratos de picão e de jabuticaba, respectivamente. Soluções: titulada, $\mathrm{NaOH} 8,3$ x $10^{-4} \mathrm{molL}^{-1}$; titulante, $\mathrm{HCl}$; volume de $\mathrm{HCl}=200 \mu \mathrm{L}$. Equações e coeficientes de correlação das respectivas curvas analíticas:

$2,1 \%$ e 3,3\%, para os extratos de picão e de jabuticaba, respectivamente, $(\mathrm{n}=10)$, os resultados obtidos usando Fenolftaleína e os extratos não diferiram a um nível de $95 \%$ de confiança, conforme descrito nas Tabelas 1 e 2 .

\section{Conclusões}

Considerando-se as facilidades de obtenção dos extratos de algumas plantas, a riqueza da nossa flora, as semelhanças entre os resultados obtidos com os extratos naturais brutos e Fenolftaleína, pode se concluir que os extratos investigados, possuem propriedades indicadoras de $\mathrm{pH}$ em potencial. Quanto a aplicação do sistema em fluxo, os resultados foram bastante satisfatórios. Portanto, tanto os extratos analisados como o sistema em fluxo, pode representar uma alternativa viável na titulação espectrofotométrica por reação de neutralização.

Tabela 1: Padronização de $\mathrm{HCl}$ com $\mathrm{NaOH} 8$ x 10-2 molL-1, usando diferentes indicadores.

\begin{tabular}{c|ccc|ccc}
\hline \multirow{2}{*}[\mathrm{Hcl}]{$_{\text {Teo }}$} & \multicolumn{3}{|c|}{ Padronização por titulação visual } & \multicolumn{3}{c}{ Padronização por titulação em fluxo } \\
\cline { 2 - 7 } & Fen & Pic & Jab & Fen & Pic & Jab \\
\hline 0,100 & $0,100 \pm 0,002$ & $0,090 \pm 0,003$ & $0,100 \pm 0,015$ & $0,090 \pm 0,000$ & $0,100 \pm 0,001$ & $0,100 \pm 0,001$ \\
0,700 & $0,680 \pm 0,012$ & $0,700 \pm 0,005$ & $0,690 \pm 0,010$ & $0,700 \pm 0,000$ & $0,710 \pm 0,000$ & $0,690 \pm 0,000$ \\
1,000 & $1,020 \pm 0,006$ & $1,040 \pm 0,009$ & $1,080 \pm 0,009$ & $1,100 \pm 0,001$ & $0,950 \pm 0,000$ & $1,130 \pm 0,001$ \\
\hline
\end{tabular}

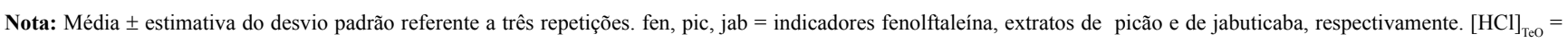
concentrações teóricas em molL ${ }^{-1}$. 
Tabela 2: Determinação da acidez em amostras de refrigerantes, suco de abacaxi e vinagre. As amostras foram tituladas com $\mathrm{NaOH}^{8,3} \mathrm{x} 10^{-4}$ molL ${ }^{-1}$. A acidez nas amostras foi expressa em molL ${ }^{-1}$.

\begin{tabular}{cccc|ccc}
\hline \multicolumn{4}{c}{ Titulação visual } & \multicolumn{3}{c}{ Titulação em fluxo } \\
\hline Amostras & Fen & Pic & Jab & Fen & Pic & Jab \\
\hline A1 & $0,016 \pm 0,001$ & $0,015 \pm 0,001$ & $0,016 \pm 0,002$ & $0,017 \pm 0,000$ & $0,019 \pm 0,000$ & $0,014 \pm 0,000$ \\
A2 & $0,025 \pm 0,001$ & $0,026 \pm 0,001$ & $0,026 \pm 0,003$ & $0,026 \pm 0,000$ & $0,028 \pm 0,000$ & $0,024 \pm 0,000$ \\
A3 & $0,032 \pm 0,000$ & $0,031 \pm 0,001$ & $0,032 \pm 0,003$ & $0,033 \pm 0,000$ & $0,036 \pm 0,000$ & $0,037 \pm 0,000$ \\
A4 & $0,031 \pm 0,001$ & $0,030 \pm 0,001$ & $0,031 \pm 0,002$ & $0,034 \pm 0,000$ & $0,036 \pm 0,000$ & $0,037 \pm 0,000$ \\
A5 & $0,025 \pm 0,001$ & $0,025 \pm 0,001$ & $0,024 \pm 0,003$ & $0,026 \pm 0,000$ & $0,023 \pm 0,000$ & $0,028 \pm 0,000$ \\
A6 & $0,170 \pm 0,000$ & $0,175 \pm 0,002$ & $0,165 \pm 0,002$ & $0,165 \pm 0,000$ & $0,180 \pm 0,000$ & $0,160 \pm 0,000$ \\
A7 & $0,630 \pm 0,001$ & $0,650 \pm 0,001$ & $0,620 \pm 0,004$ & $0,660 \pm 0,000$ & $0,680 \pm 0,000$ & $0,670 \pm 0,000$
\end{tabular}

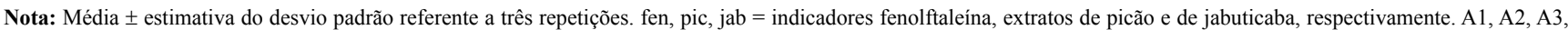
A4, A5, A6 e A7 = amostra de guaraná, pitchula limão, água tônica, sprite, soda limonada, suco de abacaxi e vinagre, respectivamente.

\section{Agradecimentos}

Os autores agradecem ao $\mathrm{CNPq}$ pelo apoio financeiro concedido.

\section{BIBLIOGRAFIA}

1. Baccan, N.; Andrade, J. C.; Godinho, O. E. S.; Barone, J. S., Química Analítica Qauntitativa Elementar, 2a ed., Ed. Unicamp: Campinas, 1979 p. 46.

2. Vogel,A. I., Análise Química Quantitativa; Mendham, J.; Denney, R. C.; Barnes, J. D.; Thomas, M. J. K.; 6a Ed.; LTC, 2000; p 87.

3. Hallikainen, K.E.; Pompeo, D.J., Instruments 1952, $25,335$.

4. Ruzicka, J.; Hansen, E.H.; Mosbaek, H., Anal. Chim. Acta 1977, 92, 235.

5. Stewart, K. K.; Rosenfeld, A. G., Anal. Chim. Acta 1982, 54, 2368.

6. Nagy, G.; Feher,, Zs.; Toth, K.; Pungor, E., Anal. Chim. Acta 1978, 100, 181.

7. Paim, A. P. S.; Kronka, E. A. M.; Reis, B. F.; Korn, M., Quím. Nova 1998, 21, 47.

8. Almuaibed, A. M.; Townshend, A., Talanta 1992, 39, 1459.
9. Sultan, M., Talanta 1993, 40, 593.

10. Alamo, J. M.; Mquieira, A.; Puchades, R.; Sagrado, S., Fresenius J. Anal. Chim. 1993, 347, 293.

11. Korn, M; Gouveia, L. F. B. P.; Oliveira, E. ; Reis B F, Anal. Chim. Acta, 1995, 313, 177.

12. Rao, G. G.; Sastry, G. S., Anal. Chim. Acta 1971, 56, 325.

13. Sultan, S. M.; Abdennabe, A. M.; Suliman, F. E. O., Talanta 1994, 41, 125.

14. Jain, A.; Chaurasia, A.; Verma, K. K., Talanta 1995 , $42,779$.

15. Nóbrega, J. A.; Lopes, G. S., Talanta 1996 43, 971.

16. Pérez-Ruíz, T.; Martinez-Losano, C.; Sanz, A., Anal. Chim. Acta 1995, 309, 299.

17. Terci, D. B. L.; Rossi, A. V., Quim. Nova 2002, 25, 684

18. García, I. L.; Vinas, P.; Campillo, N.; Córdoba, M. H., Anal. Chim. Acta 1995, 308, 67.

19. Lazaro, F.; Rios, A.; Luque de Castro, M. D.; Valcarcel, M., Analyst 1986, 111, 163.

20. Hernandez, J.; Alonso, A.; Almendral, M. J.; Garcia, C., Anal. Chim. Acta 1986, 184, 246.

21. Taylor, P. D., Talanta 1995, 42, 845. 
22. Couto, A. B. C.; Ramos, L. A.; Cavalheiro, E. T. G., Quim. Nova, 1998, 21, 221.

23. Rossi, A. V. ; Terci, D. B. L, Quím. Nova, 2002, 25, 523.

24. Cavalheiro, É. T. G.; Cortes, M. S; Ramos, L. A., Quím., 2007, 30, 1014.

\section{Jonas A. Vieira* ${ }^{*}$, Lourival \\ C. de Faria \& Wesson}

Guimarães².

${ }^{1}$ Unidade Universitária de Ciências Exatas e Tecnológicas, Universidade Estadual de Goiás, Campos Henrique Santillo, CP, 459, CEP 75001970, Anápolis-GO.

2 Instituto de Química, Universidade Federal de Goiás, Campos Samambaia, CP 131, 74001-970 - Goiânia-GO

*e-mail: jonas@ueg.br 